Article

\title{
Bimodal Benefits for Lexical Tone Recognition: An Investigation on Mandarin-speaking Preschoolers with a Cochlear Implant and a Contralateral Hearing Aid
}

\author{
Hao Zhang ${ }^{1}$, Jing Zhang ${ }^{1}$, Hongwei Ding ${ }^{1, *}$ and Yang Zhang ${ }^{2, *}$ (1) \\ 1 Speech-Language-Hearing Center, School of Foreign Languages, Shanghai Jiao Tong University, \\ Shanghai 200240, China; zhang.hao@sjtu.edu.cn (H.Z.); elainezhang@sjtu.edu.cn (J.Z.) \\ 2 Department of Speech-Language-Hearing Sciences, University of Minnesota, Minneapolis, MN 55455, USA \\ * Correspondence: hwding@sjtu.edu.cn (H.D.); zhanglab@umn.edu (Y.Z.); Tel.: +1-612-624-7878 (Y.Z.)
}

Received: 22 March 2020; Accepted: 15 April 2020; Published: 17 April 2020

check for updates

\begin{abstract}
Pitch perception is known to be difficult for individuals with cochlear implant (CI), and adding a hearing aid (HA) in the non-implanted ear is potentially beneficial. The current study aimed to investigate the bimodal benefit for lexical tone recognition in Mandarin-speaking preschoolers using a $\mathrm{CI}$ and an $\mathrm{HA}$ in opposite ears. The child participants were required to complete tone identification in quiet and in noise with $\mathrm{CI}+\mathrm{HA}$ in comparison with $\mathrm{CI}$ alone. While the bimodal listeners showed confusion between Tone 2 and Tone 3 in recognition, the additional acoustic information from the contralateral HA alleviated confusion between these two tones in quiet. Moreover, significant improvement was demonstrated in the $\mathrm{CI}+\mathrm{HA}$ condition over the $\mathrm{CI}$ alone condition in noise. The bimodal benefit for individual subjects could be predicted by the low-frequency hearing threshold of the non-implanted ear and the duration of bimodal use. The findings support the clinical practice to fit a contralateral HA in the non-implanted ear for the potential benefit in Mandarin tone recognition in CI children. The limitations call for further studies on auditory plasticity on an individual basis to gain insights on the contributing factors to the bimodal benefit or its absence.
\end{abstract}

Keywords: lexical tones; bimodal benefit; speech learning; cochlear implant (CI); hearing aid (HA)

\section{Introduction}

As a highlight of groundbreaking neural prosthesis in bioengineering, the cochlear implant (CI) provides accessibility to sound for individuals with profound hearing impairment [1,2]. The remarkable success of the $\mathrm{CI}$ is largely attributable to the proper use of signal transduction technology to harness central auditory plasticity of the implantees [2]. While CI users can significantly benefit from the neuroplasticity driven by the CI-empowered learning experience in developing their auditory, linguistic, and cognitive skills [3-9], pitch perception poses a unique challenge for these individuals. A vast body of literature demonstrated that $\mathrm{CI}$ recipients show deficits in pitch-related perceptual tasks, including voice emotion perception [10-12], speech prosody recognition [13-15], music appreciation [16-18], and lexical tone perception [19-21]. One known factor here lies in the limitations of the contemporary CI multichannel technology that encodes degraded spectral-temporal signals of the auditory input. Degradations in the representation can impair and even preclude the reception of pitch cues. The current study on Mandarin Chinese speakers aimed to investigate applicable interventions in alleviating the deficits in the representation and reception of pitch information for the pediatric CI users.

There has been a growing number of CI prescriptions not only for individuals with bilateral deafness but also for those with profound unilateral hearing loss. Some clinical practices point to 
compelling evidence in improving CI individuals' speech perception by means of bimodal hearing for unilaterally implanted candidates with some residual acoustic hearing. Bimodal hearing refers to the combination of two different solutions for hearing loss, with fitting a CI in one ear while using a hearing aid (HA) in the opposite ear. Behaviorally, successful bimodal listeners obtained an improvement in speech understanding with CI + HA stimulation (i.e., a unilateral CI together with a contralateral HA) relative to with a unilateral CI alone [22-24]. The improved perceptual outcome in the bimodal stimulation over the CI alone condition is referred to as bimodal benefit, and it has been demonstrated in a number of studies of non-tonal languages. Several research groups provided corroborating evidence for bimodal benefits with task-specific tests in the perception of segmental linguistic features that contain low-frequency components, such as voicing, semivowels, and nasals $[25,26]$, or in the perception of supra-segmental linguistic features, such as intonation, emphasis, and stress $[27,28]$. The behavioral improvements might be underpinned by the plasticity in auditory-related cortical areas as a consequence of the bimodal hearing experience. Nevertheless, the great heterogeneity of the CI population revealed a remarkable variability with respect to the degree of benefit from the bimodal stimulation. Some subjects tended to benefit significantly from the additional HA in the opposite ear, whereas others benefited less, or even not at all.

The great variability of the bimodal benefit leads to an initiative to pinpoint the contributing factors for this situation at the individual level. One important factor could be the degree of impairment in basic auditory function, which can lead to different amounts of residual hearing in the non-implanted ear [29]. For example, Most et al. [28] revealed significant negative correlations between the perception of supra-segmental features with bimodal stimulation and the unaided pure-tone average (PTA) of the non-implanted ear. However, it should be noted that the authors did not evaluate the relationship between the benefit magnitude in perceptual outcomes and the unaided PTA of the non-implanted ear directly. This might limit the power of validation on bimodal benefit for the individual participants. In another study, Zhang et al. [30] demonstrated that the amount of bimodal benefit in speech understanding was significantly correlated with audiometric thresholds of the non-implanted ear $(\mathrm{r}=-0.814)$. Nevertheless, some studies failed to find a reliable relationship between acoustic hearing thresholds and benefit magnitudes [31], which points to other possible contributing factors for bimodal benefits.

In recent years, there has been a surge in investigating the bimodal benefit on lexical tone perception in individuals from tonal languages [31-36]. Lexical tones are a distinctive feature of tonal languages, which utilize pitch variations to indicate contrasts in word meaning. For instance, Mandarin Chinese has four tones that can be combined with the same syllable to indicate different words (e.g., /ma/ could respectively mean 'mother', 'hemp', 'horse' or 'scold' depending on whether the tone is flat, rising, dipping or falling). Findings from tonal language speakers have been inconsistent. Some revealed no significant bimodal benefit on lexical tone recognition either in quiet or in noise [36]. Some reported a significant bimodal benefit for lexical tone recognition in noise but not in quiet [32,35]. Others showed a significant bimodal benefit in quiet for tonal perception tasks that depend more on pitch cues $[33,34]$. While most of the studies focused on the verification of bimodal benefits in various listening conditions, very few investigated the candidate factors that could account for the individual variability in bimodal benefit. One exception is Chang et al. [31], which reported no significant correlation between the acoustic hearing threshold of the non-implanted ear and the bimodal performance in Mandarin tone recognition. The lack of solid evidence for the underlying contributing factors of the bimodal benefit variability leaves the topic open for further inspection. In particular, there has been little work on young bimodal children who are still under a sensitive period of auditory cortical organization for tonal acquisition.

Several initiatives have been launched to investigate the lexical tone perception in Mandarin children with CIs. Based on differences in pitch height and pitch contour, Mandarin tones are traditionally classified into four categories: Tone 1 (T1) with a high-flat pitch, Tone 2 (T2) with a mid-rising pitch, Tone 3 (T3) with a falling-rising pitch, and Tone 4 (T4) with a high-falling pitch. 
Prior studies have demonstrated that T1 and T4 are significantly easier to perceive than T2 and T3 for native CI children, with more confusion between the identification of T2 and T3 [21,37]. However, it is still an open question whether additional acoustic hearing from the non-implanted ear with an HA has the potential to enhance the representation of different tone patterns and to alleviate the confusion between T2 and T3. Notably, although large individual variability was found across the studies, age at implantation was negatively correlated with tonal perceptual performance, with earlier implanted children obtaining higher perceptual scores. Age at implantation and duration of CI use have been demonstrated as two major demographic factors contributing to the variability in lexical tone perception in pediatric implantees [38], and the two factors are also recognized as significant predictors of sentence-level intelligibility [39]. Under the scope of neural plasticity, there is a critical period for cochlear implantation, with the auditory plasticity decreasing as age increases, and with the expected cortical organizing as implant experience accumulates $[1,40,41]$. Thus, the demographic factors need to be considered to explain pediatric participants' bimodal outcome (including bimodal benefit and benefit magnitude) in both benign and adverse listening conditions.

The primary objective of this study was to investigate the nature of the bimodal benefit for Mandarin tone recognition at both group and individual levels in native kindergarten-aged children who use CI in one ear and HA in the other. Three specific hypotheses were tested: (1) the child participants would show confusion between $\mathrm{T} 2$ and $\mathrm{T} 3$, which can be alleviated by bimodal hearing; (2) the noisy listening condition would exhibit more robust bimodal benefit than the quiet condition; and (3) the audiometric thresholds and the demographic factors of the participants could account for the bimodal benefit in the preschool bimodal participants. The findings of this study would contribute to our understanding and potential development of age-appropriate intervention programs for aural rehabilitation and speech intervention for the pediatric CI population of tonal language speakers.

\section{Materials and Methods}

\subsection{Participants}

Twenty native Mandarin-speaking preschoolers born with bilateral severe-to-profound hearing loss were recruited from the Shanghai Rehabilitation Center of the Deaf Children. They had no history of psychiatric disorders or brain injuries in their medical history. All the child participants were implanted unilaterally and fitted with a contralateral HA. They used the CI in conjunction with HA devices for at least $75 \%$ of waking hours (c.f., [22,28]) according to their caregivers' observation. However, 6 were removed from data analysis due to failure in completing all experimental tests. The remaining 14 preschoolers with an age range of 4.7 to 6.6 years old (mean $=5.5$ years, $\mathrm{SD}=0.5$ years; 6 females and 8 males) were included for the current report. Their demographic information along with the CI and HA details are listed in Table 1. 
Table 1. Demographic information of the participants.

\begin{tabular}{|c|c|c|c|c|c|c|c|c|c|}
\hline Subject (Sex) & $\begin{array}{c}\text { CA } \\
\text { (yrs) }\end{array}$ & CI (ear) & $\begin{array}{l}\text { Speech } \\
\text { Strategy }\end{array}$ & HA & $\begin{array}{c}\text { Age at CI } \\
\text { (yrs) }\end{array}$ & $\begin{array}{c}\text { CI Duration } \\
\text { (yrs) }\end{array}$ & $\begin{array}{c}\text { Age at } \\
\text { Bimodal (yrs) }\end{array}$ & $\begin{array}{c}\text { Bimodal } \\
\text { Duration (yrs) }\end{array}$ & $\begin{array}{c}\text { PTA } \\
\text { (dB HL) }\end{array}$ \\
\hline $\mathrm{S} 1(\mathrm{M})$ & 4.9 & OPUS2 (R) & FS4-P & Phonak Bolero Q50 & 0.9 & 4.0 & 0.9 & 4.0 & 85 \\
\hline $\mathrm{S} 2(\mathrm{M})$ & 5.9 & OPUS2 (R) & FS4-P & Widex C4-FS & 0.9 & 5.0 & 0.9 & 5.0 & 83 \\
\hline $\mathrm{S} 3(\mathrm{M})$ & 5.6 & Naida (R) & HiRes-Optima & Phonak Naida S IX & 2.7 & 2.9 & 2.7 & 2.9 & 78 \\
\hline $\mathrm{S} 4(\mathrm{M})$ & 5.3 & Nucleus6 (L) & $\mathrm{ACE}$ & ReSound AL777 & 2.7 & 2.6 & 3.6 & 1.7 & 67 \\
\hline S5 (M) & 5.7 & OPUS2 (R) & FS4-P & Widex C3-FS & 1.7 & 4.0 & 1.7 & 4.0 & 65 \\
\hline S6 (M) & 5.6 & OPUS2 (R) & FS4-P & Phonak Naida S IX & 1.0 & 4.6 & 1.0 & 4.6 & 88 \\
\hline S7 (M) & 4.8 & Nucleus6 (R) & ACE & Phonak Naida S IX & 1.5 & 3.3 & 1.5 & 3.3 & 83 \\
\hline S8 (M) & 6.6 & OPUS2 (R) & FS4-P & Widex C4-FS & 1.4 & 5.2 & 4.6 & 2.0 & 68 \\
\hline S9 (F) & 5.6 & Nucleus6 (L) & ACE & Phonak Q90 SP & 3.3 & 2.3 & 3.3 & 2.3 & 56 \\
\hline $\mathrm{S} 10(\mathrm{~F})$ & 5.4 & Nucleus6 (R) & $\mathrm{ACE}$ & Phonak Naida S IX & 3.0 & 2.4 & 3.7 & 1.7 & 92 \\
\hline S11 (F) & 6.2 & Nucleus5 (R) & $\mathrm{ACE}$ & Phonak Naida S IX & 1.1 & 5.1 & 1.2 & 5.0 & 63 \\
\hline S12 (F) & 5.5 & Nucleus6 (R) & $\mathrm{ACE}$ & Phonak SKY Q90-RIC & 2.8 & 2.7 & 2.8 & 2.7 & 60 \\
\hline $\mathrm{S} 13(\mathrm{~F})$ & 5.8 & Nucleus5 (L) & ACE & Phonak Q90 UP & 3.2 & 2.6 & 3.3 & 2.5 & 77 \\
\hline S14 (F) & 4.7 & Freedom (R) & $\mathrm{ACE}$ & Phonak Naida S V SP & 1.2 & 3.5 & 1.2 & 3.5 & 83 \\
\hline
\end{tabular}

CA: chronological age; CI: cochlear implant; HA: hearing aid; yrs: years; PTA: unaided three-frequency pure-tone average at 125, 250, and 500Hz for the non-implanted ear. 
Standard audiometric assessments for pure tones from $125 \mathrm{~Hz}$ to $8 \mathrm{kHz}$ were performed to determine the hearing impairment of each child participant. Figure 1 and Supplementary Table S1 show the participants' unaided and aided hearing thresholds in the non-implanted ear at each frequency. The PTA was calculated for each participant based on the average of hearing thresholds at three low frequencies, 125, 250, and $500 \mathrm{~Hz}$, in the better ear (i.e., the non-implanted ear for the current participants). This approach was adopted in previous studies to examine the relationship between audiometric thresholds and pitch-related speech and music perception in bimodal subjects [42]. In addition, we tested nonverbal intelligence with the Hiskey-Nebraska test of learning aptitude (HNTLA) [43]. All participants scored significantly higher than the passing criteria of 84 for normal nonverbal intelligence. This study was approved by the Ethics Committee of School of Foreign Languages, Shanghai Jiao Tong University. Informed consent was received from the caregiver of each child participant.

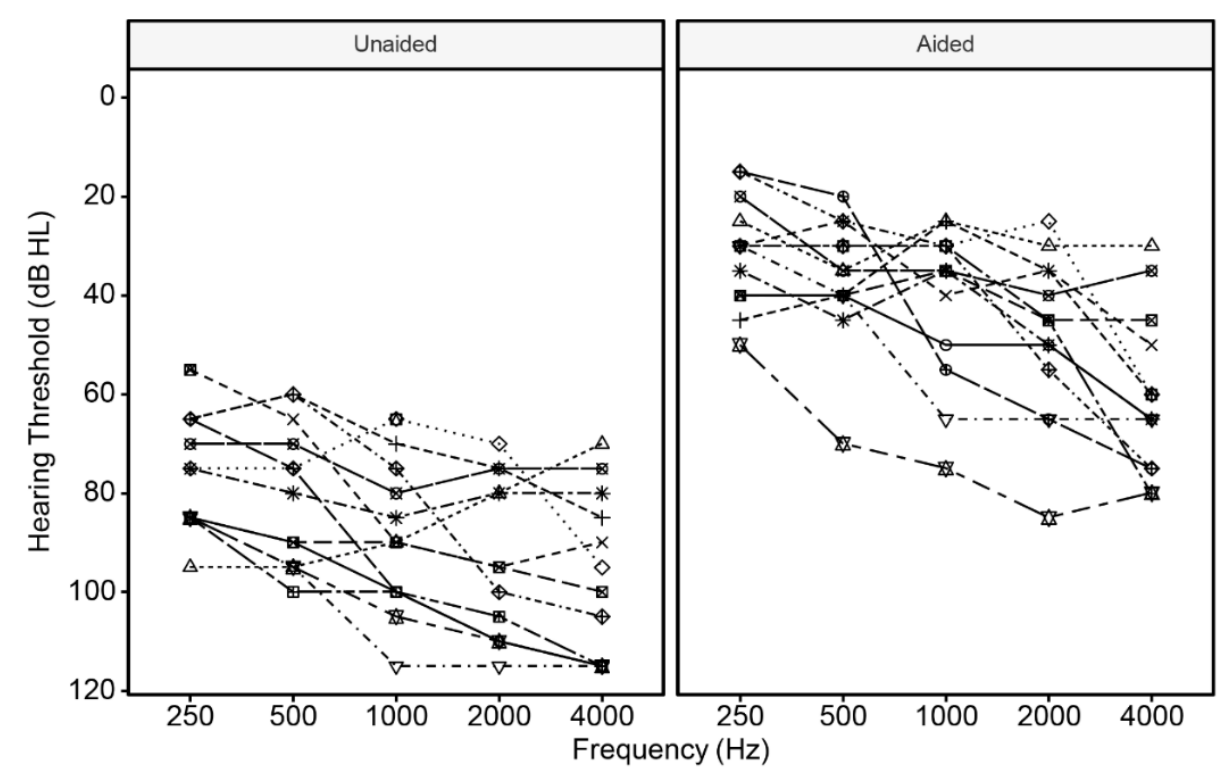

Figure 1. Unaided and aided hearing thresholds for the non-implanted ear of each child participant.

\subsection{Stimuli}

Stimuli in the speech recognition task were the four Mandarin tones naturally produced with the /i/ syllable. They were recorded from four native Mandarin-speaking adults ( 2 males and 2 females) in a double-walled, sound-attenuated booth. Each speaker was required to produce each tone 10 times. The speech samples were recorded at a sampling rate of $44.1 \mathrm{kHz}$ (16 bit). An experienced phonetician selected five speech samples for each tone per speaker, resulting in 80 test stimuli in total. All stimuli were normalized with equal average root-mean-square (RMS) intensity level. The average duration of the T1, T2, T3, and T4 stimuli are 537, 532, 614, and 374 milliseconds (ms), respectively. The pitch contours of the four tones were extracted from the test stimuli using Praat [44], which are depicted in Figure 2 based on speaker genders. The average pitch ranges of T1, T2, T3, and T4 stimuli are 20, 62, 50 , and $82 \mathrm{~Hz}$ for the male speakers, respectively. By contrast, the accurate pitch ranges are 45, 90, 74, and $122 \mathrm{~Hz}$ for the female speakers, respectively, for the four tone types (see Supplementary Table S2 for detailed information). 


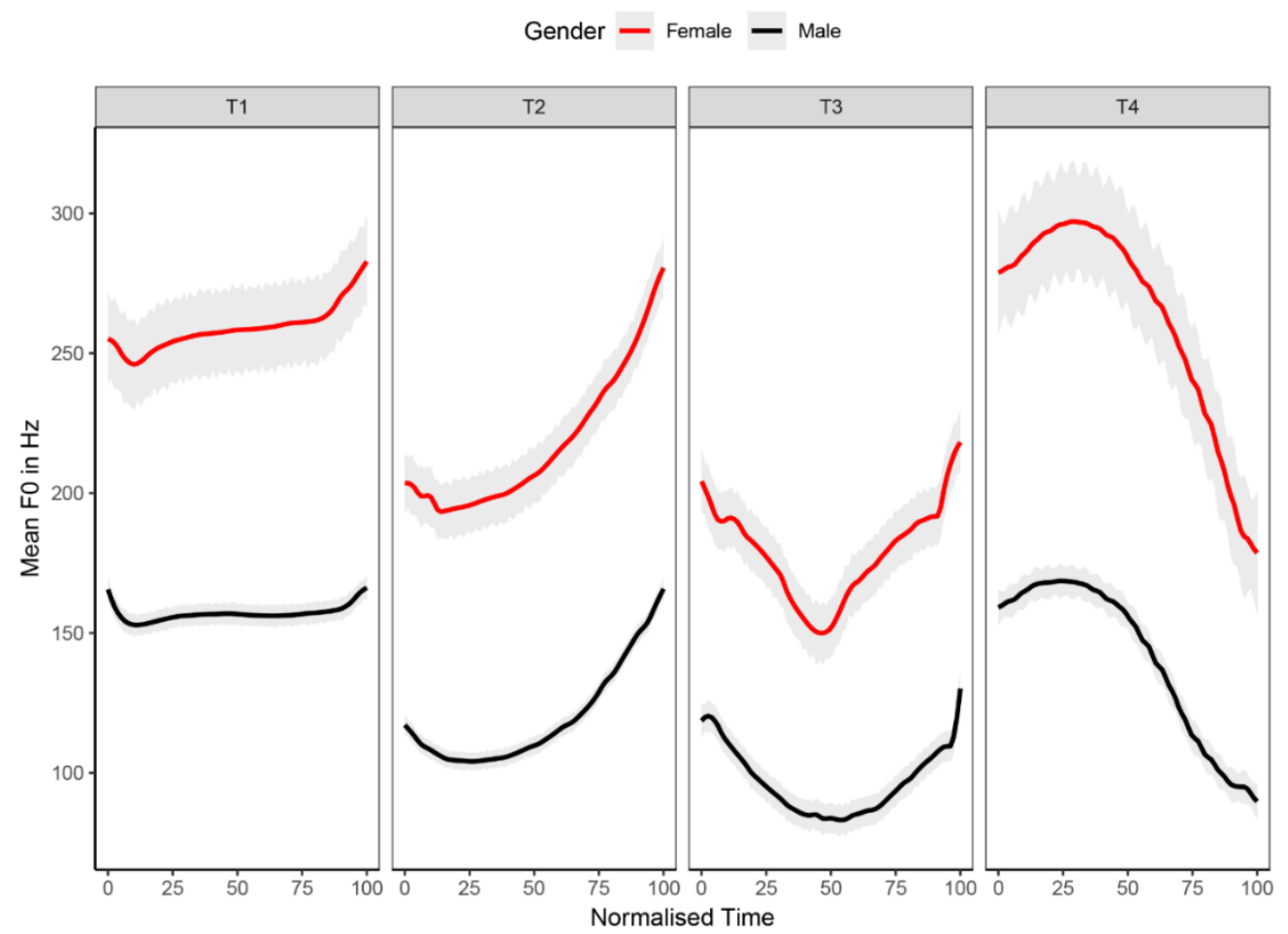

Figure 2. Pitch contours of the lexical tone recognition materials. Grey shades indicate standard error.

The lexical tone recognition test was performed in both quiet and noisy listening conditions. To this end, the speech spectrum-shaped noise (SSN) was used as a masker to corrupt the test stimuli at the signal-to-noise ratio (SNR) level of $+5 \mathrm{~dB}$. The SSN was generated by passing white noise through a finite impulse response filter derived from the average spectrum of all the speech samples (c.f., [45]). Our pilot test with four SNR levels (i.e., $+10,+5,+3$, and $0 \mathrm{~dB}$ ) in five subjects indicated that the $+5 \mathrm{~dB}$ SNR would be appropriate to avoid both ceiling and floor effects. Previous research had also used the SNR of $+5 \mathrm{~dB}$ in word and sentence recognition tests for CI participants (e.g., [22,32,46]).

\subsection{Procedures}

All child participants performed the test using their own $\mathrm{CI} / \mathrm{HA}$ devices and daily settings for volume and sensitivity in a sound-treated therapy room. The preschoolers' lexical tone recognition was examined in two device conditions and two listening conditions. The order of the two device conditions was randomized and counterbalanced across all participants. In order to reduce the practice and fatigue effects, the measure under different device conditions was performed on two lab visits with a one-week separation.

The test was implemented with E-Prime 2.0 on a Windows-based laptop. The auditory stimuli were delivered in the free field via a loudspeaker (JBL CM220) which was placed approximately $1.2 \mathrm{~m}$ from the front of the listener. The stimuli were calibrated to be at a sound level of approximately $65 \mathrm{~dB}$ SPL to the location of the child participant's head. Before the test session, a brief instructional session with practice was provided to ensure that all participants could follow the requirements of the task. The child participants were instructed to perform sound-picture matching with each picture representing the pitch contour of the corresponding tone, from the four options for the four Mandarin tones presented on the laptop computer screen. The four pictures of driving cars are popular materials for teaching the four Chinese lexical tone categories in formal school settings, with a car driving on a level road indicating T1, a car driving on a road with rising slope indicating T2, a car driving on a road with falling and rising slopes indicating $\mathrm{T} 3$, and a car driving on a road with a falling slope indicating T4. The experimenter presented each tone with the corresponding picture to explain the relationship 
for the sound-picture matching to the participant. A practice block was then offered with trial-by-trial feedback. To avoid carryover, the stimuli for the demonstration and practice were not included for the following test. Only after the participant consecutively succeeded in matching the four tone types with the correct pictures in a random order, would she/he be allowed to proceed to the test.

The testing session adopted a four-alternative, forced-choice (4 AFC) paradigm for each stimulus. The preschoolers were required to pay attention to the presented stimulus and encouraged to imitate the sound while pointing to the correct picture. If the vocal response contradicted with their picture pointing, they would be required to clarify their choice. This stipulation allowed the experimenter to double check the participants' intended response. The child participants were encouraged to make a guess when they were uncertain about the choice. Their trial-by-trial responses were logged by the experimenter via pressing the corresponding keys on the keyboard. The total of 80 tonal stimuli were randomly presented to each child within two blocks. Both tone types and speakers were balanced across the two blocks. Participants had a two-minute break between blocks.

\subsection{Data Analysis}

\subsubsection{Accuracy Score and Confusion Matrix}

The percentage of accuracy scores and confusion matrices of the four tones were computed for each participant in the two device conditions and the two listening conditions. The percentage score of tone recognition was transformed into a rationalized arcsine unit (RAU) score [47] for statistical analysis. The RAU score was widely used in speech intelligibility tasks to reduce saturation effect and restore homoscedasticity [48].

All statistical analyses were performed with the open source R platform (Version 3.6.0). Linear mixed-effects (LME) models were constructed to evaluate the accuracy scores of lexical tone recognition in quiet and noise for the bimodal participants. The package of lme4 [49] was used to create the LME models. As for fixed effects, tone type (i.e., T1, T2, T3, and T4), device condition (i.e., CI alone and CI + HA), listening condition (i.e., quiet and noise), and all possible interactions were treated as fixed factors. In addition, speaker gender was treated as a control factor, since the intrinsic differences in voice pitch between male and female speakers (see Figure 2) could potentially affect the preschool participants' lexical tone recognition. The participant was treated as a random effects variable. The full model with all fixed factors was compared with a simplified model that excluded a fixed factor in question using the ANOVA function in lmerTest package [50]. The Akaike information criterion (AIC) was computed to check whether the fixed factor in question could contribute to an improved fit for the constructed model. Eventually, the model with the lowest AIC was selected to estimate the significance of the fixed factors, with $\alpha$ setting at 0.05. In addition, the ANOVA function was used to obtain $F$ - and $p$-values of the significant fixed factors, which uses the Satterthwaite approximation for the degree of freedom. Post-hoc pairwise comparisons were performed for the significant fixed factors using the lsmeans package [51] with the Bonferroni adjustment to obtain $t$-ratios, and $p$-values.

Beyond the accuracy scores for tone recognition, we further analysed the error patterns for each device condition in the two listening conditions. Specifically, in order to investigate the confusion between T2 and T3 across the child participants, the error scores with T2 misidentified as T3 and T3 misidentified as T2 were estimated for each participant in each device condition and listening condition. An additional LME model was created to examine the significance of tone type (i.e., T2 and T3), device condition, and listening condition on the misidentified error scores, using by-participant random effects.

\subsubsection{Normalized Bimodal Benefit Score}

Bimodal benefit is traditionally calculated as the difference in perceptual performance between the $\mathrm{CI}+\mathrm{HA}$ device condition and CI alone condition. One shortcoming of this absolute calculation, as specified by Zhang et al. [30], is the failure of taking into account the limited improvement room when 
the participant obtained a high level of perceptual performance in CI alone condition. For example, an improvement of $10 \%$ absolute score would be different for a participant obtained from $60 \%$ accuracy in $\mathrm{CI}$ alone condition to $70 \%$ accuracy in $\mathrm{CI}+\mathrm{HA}$ condition, relative to another participant who improved from $90 \%$ to $100 \%$ ceiling level. In the first case with a starting score of $60 \%$, there is $40 \%$ of room for improvement. In the second case with a $90 \%$ starting score, however, there is only $10 \%$ room to reach ceiling-level performance. To handle such inter-subject differences, the normalized benefit score was calculated according to the formula (i.e., $100 \times(($ Bimodal - CI alone $) /(100 \%-C I$ alone $)))$ as defined specifically in Zhang et al. [30]. In addition, for child participants who achieved lower accuracy score in bimodal condition than in CI alone condition, the formula was reworked (i.e., $100 \times(($ Bimodal - CI alone) / (CI alone))) to describe the potential drop in lexical tone recognition with an additional HA (c.f., [30]). The normalized formula could control the different performances in CI alone condition into a comparable level across individuals, which contributes to an indexed score ranging from -100 (for extreme cases drop from $100 \%$ in CI alone condition to $0 \%$ in bimodal condition) to +100 (for extreme cases improve from $0 \%$ in CI alone condition to $100 \%$ in bimodal condition). In the field of speech and hearing sciences, this normalized equation has been widely adopted in recent studies to evaluate individual's bimodal benefits in speech intelligibility tasks (e.g., [46,52-57]).

\subsubsection{Regression Models for Delineating Relationships}

The hypothesized predictors for tonal recognition performance consisted of audiometric thresholds and demographic factors. The evaluations of perceptual performance of lexical tones in the current report included (1) lexical tone recognition in $\mathrm{CI}$ alone condition, (2) lexical tone recognition in $\mathrm{CI}+\mathrm{HA}$ condition, and (3) normalized benefit score for lexical tone recognition. Audiometric thresholds included (1) three-low-frequency PTA, (2) five-frequencies unaided hearing threshold, and (3) five-frequencies aided hearing threshold. The key demographic factors were (1) chronological age, (2) implanted age, (3) duration of CI use, and (4) duration of bimodal use. Multivariate regression models were constructed in $\mathrm{R}$ to assess the significant variables that contributed to the child participants' lexical tone performance and the potential bimodal benefit. Separate models were developed for the three estimates of perceptual performance with the audiometric variables and demographic variables added as fixed effects. F-statistics, and $p$-values for the fixed effects were assessed with significance level $\alpha=0.05$.

\section{Results}

\subsection{Lexical Tone Recognition}

Group mean accuracy data for the four lexical tones are shown in Figure 3, contrasting performance for the two device conditions in quiet and in noise. The average RAU scores of tonal identification for $\mathrm{CI}$ alone and CI + HA device conditions were, respectively, $93.95(S D=11.09)$ and $95.04(S D=11.64)$ in quiet, and $61.76(S D=17.91)$ and $69.17(S D=16.62)$ in noise. In quiet, both T1 and T4 obtained nearly perfect scores whereas T2 was mainly misidentified as T3 (accounting for $88.54 \%$ of all errors), and T3 was mainly misidentified as T2 (accounting for $87.28 \%$ of all errors). In noise, T4 was identified with the fewest errors, and was relatively evenly misidentified as the other three tones. However, T1 was mainly misidentified as T2 (accounting for $49.76 \%$ of errors), T2 was mainly misidentified as T3 (accounting for $48.21 \%$ of errors), and T3 was mainly misidentified as T2 (accounting for $56.17 \%$ of errors). The results demonstrated the confusion between T2 and T3 identification both in quiet and in noise. See Supplementary Table S3 and Table S4 for detailed information of the confusion matrices of the four tones in quiet and noise. 


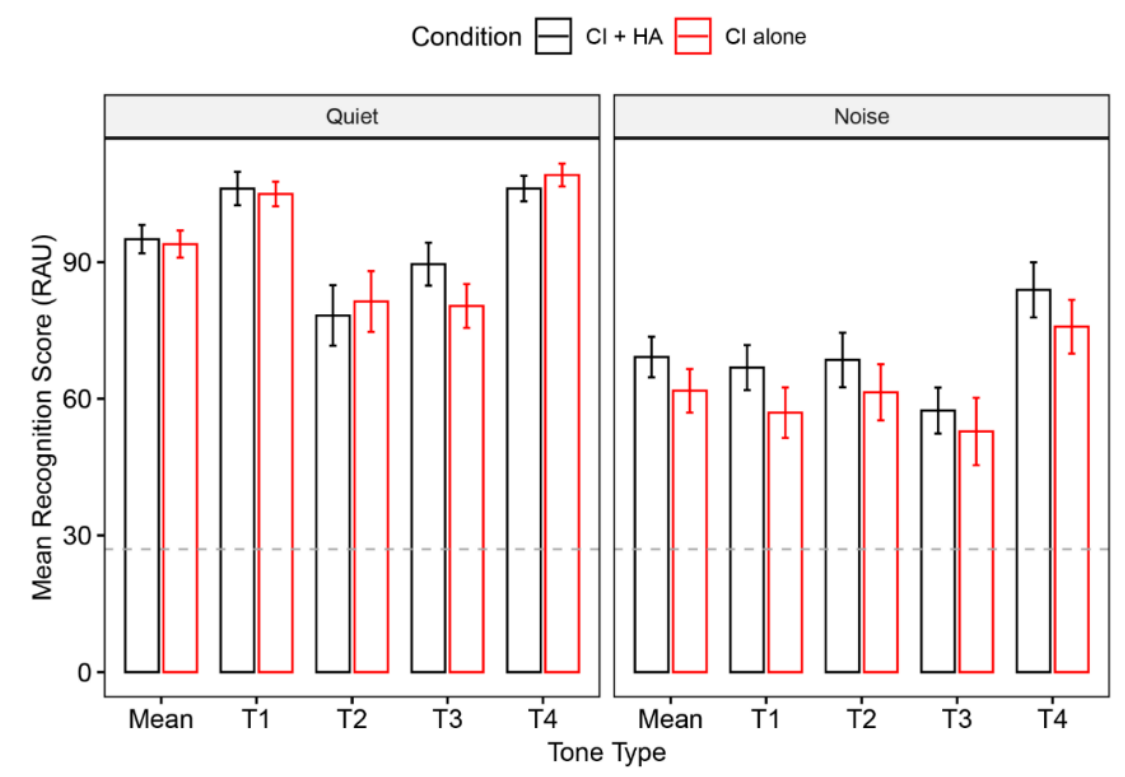

Figure 3. Mean recognition scores in rationalized arcsine unit (RAU) of each tone type in quiet and noise.

The dash lines indicate the chance level. Error bars represent the standard errors across all participants.

Statistical analysis for the accuracy data revealed a significant main effect of device condition $(F=5.96, p=0.02)$. Compared with $C I$ alone, lexical tones were overall significantly better recognized with bimodal stimulation $(t=2.41, p=0.03)$. The significant main effect of tone type $(F=12.99$, $p<0.001$ ) was also revealed, with the identification of T4 significantly better than all other three tones (all $t s>3.19, p s<0.05$ ). Moreover, there were also significant interaction effects of device condition by listening condition $(F=4.14, p=0.04)$. Further post-hoc comparisons in quiet revealed no significant difference on lexical tone recognition between the two device conditions, with only T3 showing marginally better $\mathrm{CI}+\mathrm{HA}$ performance than with $\mathrm{CI}$ alone $(t=1.85, p=0.06)$. By contrast, in noisy listening condition, the perceptual performance of lexical tones was significantly better in bimodal hearing than in $\mathrm{CI}$ alone condition $(t=3.13, p=0.003)$.

Additional LME models for error analysis found a significant three-way interaction of tone type by listening condition by device condition $(F=4.64, p=0.03)$. Post-hoc pairwise comparisons with Bonferroni adjustment showed that T3 was less misidentified as T2 for CI + HA condition relative to CI alone condition in quiet $(t=-2.06, p=0.04)$, but not in noise $(p=0.97)$.

The individual listeners' results are displayed in Figure 4. Paired-samples T tests were conducted to compare the perceptual performance of each individual between $\mathrm{CI}$ alone and CI + HA device conditions. In quiet, no subject showed a significant difference between the two device conditions (all $p \mathrm{~s}>0.08)$. However, in noise, a significant bimodal benefit was revealed for S13 $(t=3.69, p=0.005)$, and S14 $(t=4.1, p=0.003)$. 


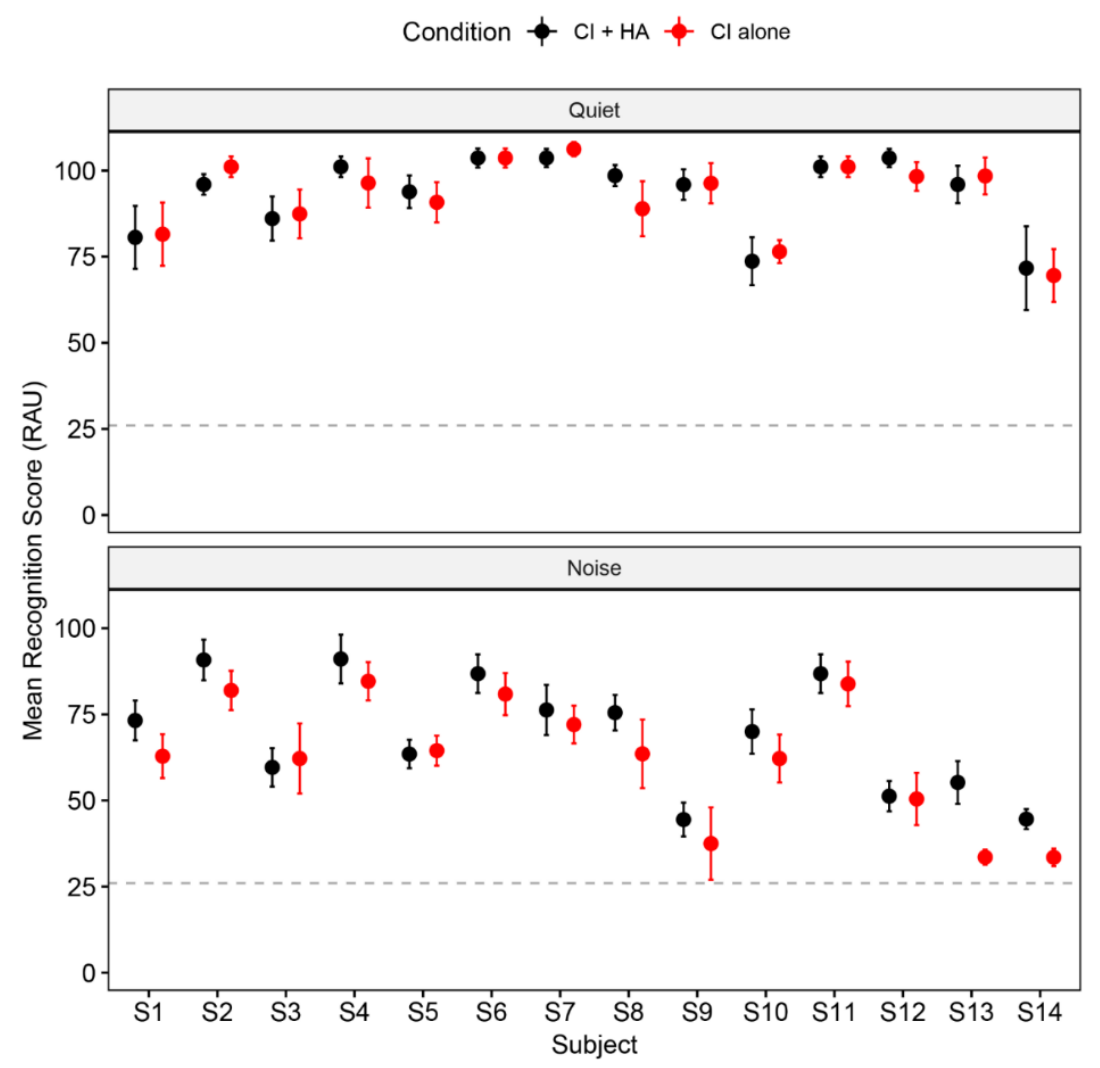

Figure 4. Mean RAU scores of natural tone recognition for each participant in quiet and noise. Dash lines indicate the chance level. Error bars represent the standard errors across four tone types.

\subsection{Normalized Bimodal Benefit}

To account for individual differences in performance, the normalized benefit score compares the two device conditions and computes relative gain in the bimodal condition over the $\mathrm{CI}$ alone condition for each individual. On average, the normalized benefit score on lexical tone recognition was +16.64 (ranging from -6.35 to +69.23 ) in quiet and +17.64 (ranging from -1.89 to +34.55 ) in noise. Five children had negative scores in quiet condition, suggesting the lack of bimodal benefit $(p=0.42$, exact binomial test). In comparison, 12 children showed positive scores in the noisy condition, indicating the tangible bimodal benefits ( $p<0.01$, exact binomial test). The mean normalized benefit score was calculated as the average score of the two listening conditions for each participant. The average score was +17.14 on group level, ranging from 0 to +50.69 . The normalized benefit scores for each child participant in quiet and noise are shown in Figure 5. 


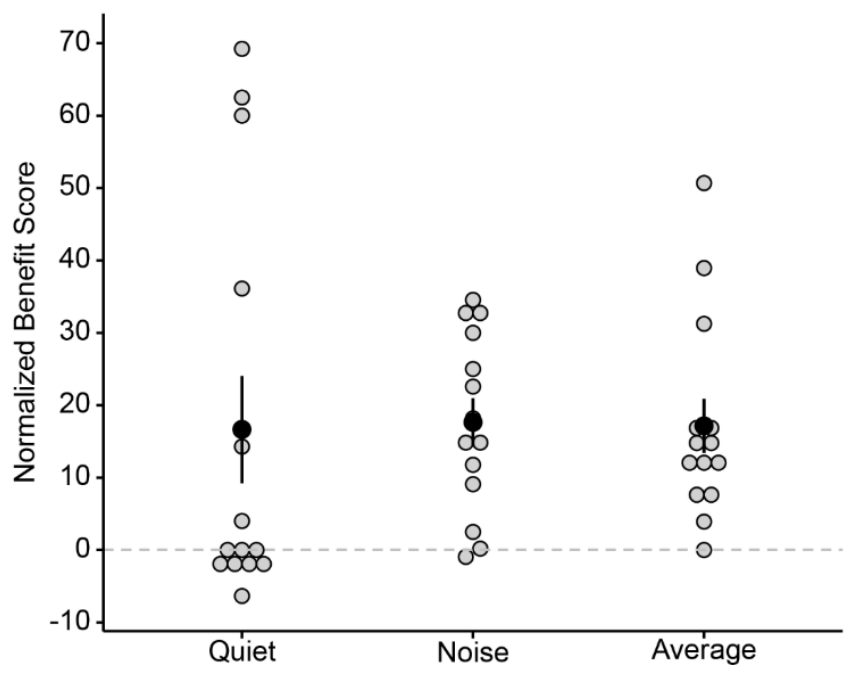

Figure 5. Normalized benefit score received from an additional HA in the non-implanted ear for lexical tone recognition in quiet (left column), in noise (middle column), and the average across the two listening conditions (right column).

\subsection{Regression Analysis Results}

For the $\mathrm{CI}$ alone condition, the linear regression models revealed that $\mathrm{CI}$ duration was marginally associated with lexical tone recognition across listening conditions $(F=3.65, p=0.08)$. Specifically, CI duration was a significant predictor for lexical tone recognition in noise $(F=4.89, p=0.047)$, with increased CI use contributing to a higher accuracy score of tonal recognition in noise, but not in the quiet condition $(p=0.45)$. Similar results were obtained for the CI + HA condition. CI duration was significantly correlated with lexical tone recognition in noise $(F=5.59, p=0.036)$, but not in quiet $(p=0.4)$.

The duration of bimodal use was a significant predictor for the bimodal benefit in lexical tone recognition across the two listening conditions $(F=5.73, p=0.034)$. Overall, the normalized benefit scores improved as a function of increase in the combined use of CI and HA. Moreover, the PTA at three low frequencies in the non-implanted ear was significantly correlated with bimodal benefit in quiet $(F=8.09, p=0.015)$, with a lower PTA resulting in a higher normalized benefit score. A visual inspection of residual plots revealed that the residuals were normally distributed without any obvious deviations from homoscedasticity for each model.

\section{Discussion}

This study investigated the bimodal benefit for Mandarin tone recognition in native kindergarten-aged children who used a CI in one ear and an HA in the opposite ear. Both quiet and noisy listening conditions were tested. The main results were in line with the hypotheses. The additional HA in the non-implanted ear helped alleviate the recognition confusion between T2 and T3 in quiet. Meanwhile, significant bimodal benefits were found for tone recognition in noise. Furthermore, PTA at three low frequencies in the non-implanted ear and the duration of bimodal use were two significant predictors for the potential bimodal benefit in lexical tone recognition.

\subsection{Bimodal Benefits for Lexical Tone Recognition}

Significant bimodal benefits for lexical tone recognition were shown in noisy listening condition, and only limited benefits were exhibited in quiet with additional acoustic information from the contralateral HA alleviating the confusion between $\mathrm{T} 2$ and $\mathrm{T} 3$. The findings were consistent with previous studies on adult bimodal listeners $[32,35]$, which revealed tangible bimodal benefits in tonal identification in noise but not in quiet. 
The error pattern analysis in this study revealed that T2 and T3 were mainly misidentified as each other. The confusion between T2 and T3 was in line with prior reports that examined pediatric Mandarin-speaking CI users' lexical tone perception in quiet [21,37]. One known source for the confusion between Mandarin T2 and T3 is the acoustic similarity of the two tones [58]. As illustrated in Figure 2, T2 and T3 in isolated speech both have a rising component, and the reliable contrastive feature to discern $\mathrm{T} 3$ from $\mathrm{T} 2$ is the timing of the inflection point that turns the pitch contour from falling to rising. Given the device-related limitations of CIs in encoding pitch cues, it is reasonable to expect that an additional HA in the non-implanted ear could complement the pitch deficits and reflect the subtle differences between the two tones. Additionally, the enriched phonetic signals and the enhanced tonal distinctiveness could serve as the physical agents of neural plasticity [59], which could be conducive to auditory plasticity for acquiring distinct tonal categories. Taken together, bimodal hearing has the potential to alleviate the confusion between $\mathrm{T} 2$ and $\mathrm{T} 3$, with better tonal representations retrieved through the bimodal stimulation. Our results from the quiet listening condition provided modest support for the hypothesis. The accuracy score analysis revealed a marginally significant improvement $(p=0.06)$ for T3 identification in bimodal condition over the CI alone condition. Meanwhile, the error score analysis showed a significant decrease $(p=0.04)$ in misidentifying T3 as T2 when an additional HA was used in the non-implanted ear.

One apparent effect of noise on tone recognition in the present study was the significant drop of accuracy across all tone types, which enlarged the room for potential observable bimodal benefits. The accuracy results confirmed a significant bimodal benefit for the lexical tone recognition in noise at the group level. Individual-level examination revealed the majority of the preschoolers (12 out of 14) gained evident bimodal benefit, and two showed significant improvement for bimodal condition over CI alone condition (see Figures 4 and 5).

Which binaural mechanisms may underlie the bimodal benefit in the noisy listening condition? One plausible account is an improved pitch representation from the bilateral bimodal hearing $[29,60,61]$. Combining the degraded pitch information from the electric stimulation with the complementary low-frequency cue from the acoustic stimulation provides the bimodal listeners with better pitch representations. With the enhanced pitch representations, the child participants should presumably be capable of segregating target tones from SSN maskers, which could eventually contribute to the improved lexical tone recognition in noise with bimodal hearing. An alternative explanation is that 'glimpsing cues' may also play an important role in the bimodal benefit in noise [62-64]. According to Cooke [65], glimpses are spectral-temporal regions that are least affected by the background noise, and listeners can take advantages of glimpses in perceiving noisy speech. The spectral-temporal region refined by the low-frequency phonetic information from the additional HA might constitute useful glimpsing cues for the bimodal participants to fuse pieces of critical message as acoustic landmarks to 'bootstrap' lexical tone identification. In addition, from the perspective of neuroplasticity, bilateral bimodal hearing could prevent aural preference for the implanted ear and promote the cortical integration of input from bilateral auditory pathways [66,67]. Recently, Polonenko et al. $[67,68]$ demonstrated that children with asymmetric hearing loss, who received bimodal input with limited delay (i.e., restricting the duration of unilateral hearing), could reverse abnormal cortical aural preference and recover typical bilateral representations from both ears. Therefore, bimodal hearing is expected to improve speech detection in the noisy condition by providing bilateral representations that are retrieved from sound input from both ears.

\subsection{Contributing Variables to Bimodal Benefits}

Our data showed that both low-frequency PTA in the non-implanted ear and the duration of bimodal configuration for the participants were significant predictors for the potential bimodal benefit in their Mandarin tone recognition. The association between low-frequency PTA and bimodal benefit has been previously reported [30]. In particular, Zhang et al. [30] examined the relationship between the audiometric threshold $(\leq 750 \mathrm{~Hz})$ and bimodal benefit with Pearson correlation analyses. Our 
linear regression analysis incorporated fixed-effect and random-effect variables to better account for between-subject baseline differences and correlational structure among multiple predictor variables [69]. The results confirmed that the bimodal benefit in lexical tone recognition could be predicted by the low-frequency PTA in the non-implanted ear. The findings were compatible with a recent electroencephalography investigation that revealed bimodal hearing might be more effective in restoring typical cortical organization in children with sufficient acoustic hearing in the non-implanted ear [67].

Furthermore, one novel finding from our multivariate regression analysis is that the duration of bimodal device use is a significant contributor to the bimodal benefit in the child participants. Although previous studies have examined whether the demographic factors are able to predict CI children's lexical tone perception [38], no prior studies have reported whether age at bimodal configuration and duration of bimodal hearing are predictive of the bimodal benefit in their tonal performance. Given the critical period of auditory neuronal development and the significance of language exposure for the neural plasticity in speech learning [1,59], it is reasonable to postulate that the early bimodal adoption and the accumulating bimodal experience might contribute to the degree of bimodal benefit. Actually, our data provided the first evidence that the longer use of bimodal configuration might contribute to the development of the binaural ability to integrate acoustic and electric stimulation in the different ears, which could lead to the enhancement of bimodal benefit.

However, it should be noted that some studies failed to show consistent results. For example, Pearson correlation analyses by Chang et al. [31] did not show any significant correlations between bimodal benefit in Mandarin tone recognition and audiometric thresholds in the non-implanted ear or demographic factors of the participants. In addition to differences in statistical modelling, one noticeable difference between Chang et al.'s study and the current report lies in the methodology in calculating bimodal benefit. Chang et al.'s measure had a possible range of 0 to 100 whereas our index score permitted a wider range of -100 to 100 .

\subsection{Limitations and Implications}

One main limitation of the present study is the adoption of only one SSN masker with a fixed SNR of $5 \mathrm{~dB}$. The SSN is believed to introduce mostly energetic masking, whereas other types of maskers such as competing speech can induce both energetic and informational masking [70]. It is of great interest to investigate the effect of masking types in the recognition of Mandarin tones for native young children with congenital hearing loss. Meanwhile, our findings revealed considerable variability in lexical tone recognition with $5 \mathrm{~dB}$ SSN masker in the full range of near-ceiling level performance in some participants and floor level outcome in some others (see Figure 4). Both ceiling and floor effects limit the degree of bimodal benefit. Therefore, an individualized SNR with moderate speech reception threshold (e.g., $[42,46,61]$ ) would be an alternative approach in future investigations on the bimodal benefit for lexical tone perception.

The second limitation lies in the relatively high participant heterogeneity and small subject sample, with only six girls and eight boys. This confined our foray into examining the potential gender differences in the bimodal benefits for lexical tone recognition. To obtain robust statistical results, a much larger sample size with better-controlled demographic characteristics is desirable. In addition, although all child participants in our study were reported to be full-time users of CI devices and over $75 \%$ waking-hour users of HA devices, it should be acknowledged that the reports were based on their caregivers' estimation. Wearing time of the devices has a direct impact on the perceptual performance of the bimodal listeners [46], and not all bimodal recipients adapt well to their contralateral HA [71]. Therefore, further research is needed with proper measures of actual device wearing time and the integration ability of bimodal stimulation among the listeners.

The third limitation is in regards to the proper method for assessing bimodal benefits with the baseline performance taken into account. Although recent years have witnessed a growing trend of using the normalized equation in estimating the benefit magnitude of each individual $[30,46,52-57]$, this 
calculation method could run the risk of artificially inflating the magnitude of the bimodal benefit when the baseline performance for comparison is close to the ceiling level. Thus due caution is necessary to interpret the bimodal benefits as reported in our study and others. It would be important to develop an optimal protocol with a standardized scoring method to take into account the starting level of performance in the $\mathrm{CI}$ alone condition and avoid the potential inflation for the benefit magnitude.

Due to time consideration for feasible test protocols with children in a laboratory setting, the current study was restricted to testing the lexical tones in the /i/ syllable in quiet and in noise. Thus, further validation efforts are needed to test the generalizability of the bimodal benefits for lexical tone perception with other types of stimuli including other syllable, words and sentences as the listener would encounter in the real world.

Despite these inherent limitations, the present findings have practical implications for intervention. Overall, consistent with the majority of studies on non-tonal language speakers, our findings lend support to the clinical trial of fitting a contralateral HA in the non-implanted ear for the potential bimodal benefit in lexical tone perception for kindergarten-aged children with CIs. As a tonal language, Mandarin poses a great reliance on the pitch information for speech perception. Given the fact that an HA may provide more fine-grained low-frequency signals than a CI to improve the pitch representation, bimodal configuration can be a preferable alternative to a second CI basing on the residual acoustic hearing of the recipient's non-implanted ear. Therefore, the potential bimodal benefit should be taken into consideration in the clinical audiologic assessment for bilateral CIs in Mandarin-speaking candidates. A second CI can be recommended in the absence of bimodal benefit in quiet as well as in noise, which can provide empirical support for evidenced-based clinical practice.

The auditory rehabilitation and cochlear implantation programs in China have experienced considerable advances in the past decades. The CI candidacy criteria have extended to include individuals with a severe hearing loss. As a result, more CI candidates have aidable acoustic hearing in the non-implanted ear. The residual acoustic hearing, although it has limited use on its own, is demonstrated as providing a substantial benefit in speech perception when combined with the CI stimulation (e.g., [22,62]). The residual acoustic hearing should be taken into consideration for these individuals while setting up intervention regimens. Training studies could be designed with computer-based intervention $[6,72]$ to manipulate the speech input parameters for maximizing bimodal learning.

\section{Conclusions}

Findings of the present study confirmed a confusion pattern for T2 and T3 in Mandarin preschoolers using a CI and an HA in opposite ears. The additional low-frequency acoustic information from the HA in the non-implanted ear can alleviate the confusion between T2 and T3 in quiet, and contribute to an improvement in overall performance of lexical tone recognition in noise. Moreover, audiometric thresholds of PTA at low frequencies $(125,250$, and $500 \mathrm{~Hz})$ and bimodal duration were two significant predictors for the varying degrees of bimodal benefit in lexical tone recognition. These results provide evidence for the practice of fitting an HA in the non-implanted ear to take advantage of the potential bimodal benefit to facilitate speech learning in kindergarten-aged children with a unilateral CI. Further research is needed to examine auditory plasticity on an individual basis to gain insights on the contributing factors to the bimodal benefit or its absence in quiet and in noise.

Supplementary Materials: The following are available online at http://www.mdpi.com/2076-3425/10/4/238/s1, Table S1: Unaided and aided hearing thresholds for the non-implanted ear of the preschool participants; Table S2: Pitch contour information of each tone type; Table S3: Confusion matrix of lexical tone recognition in quiet; Table S4: Confusion matrix of lexical tone recognition in noise.

Author Contributions: H.D., Y.Z., and H.Z. conceived and designed the experiment. H.Z. and J.Z. implemented the experiment, collected and analyzed the data. H.D. and Y.Z. interpreted the data. H.Z., H.D., and Y.Z. wrote the manuscript. H.D. and Y.Z. contributed equally to this work. All authors have read and agreed to the published version of the manuscript. 
Funding: This research was funded by the Major Program of National Social Science Foundation of China, grant number 18ZDA293. Yang Zhang received additional support from the University of Minnesota's Grand Challenges Exploratory Research Grant and Brain Imaging Grant for international collaboration.

Acknowledgments: We thank the Shanghai Rehabilitation Center of the Deaf Children for the cooperation and assistance in implementing this study.

Conflicts of Interest: The authors declare no conflict of interest.

\section{References}

1. Kral, A.; Dorman, M.F.; Wilson, B.S. Neuronal development of hearing and language: Cochlear implants and critical periods. Annu. Rev. Neurosci. 2019, 42, 47-65. [CrossRef] [PubMed]

2. Moore, D.R.; Shannon, R. V Beyond cochlear implants: Awakening the deafened brain. Nat. Neurosci. 2009, 12, 686-691. [CrossRef] [PubMed]

3. Kral, A.; Sharma, A. Developmental neuroplasticity after cochlear implantation. Trends Neurosci. 2012, 35, 111-122. [CrossRef] [PubMed]

4. van Wieringen, A.; Wouters, J. What can we expect of normally-Developing children implanted at a young age with respect to their auditory, linguistic and cognitive skills? Hear. Res. 2015, 322, 171-179. [CrossRef] [PubMed]

5. Fu, Q.-J.; Galvin, J.J., III. Perceptual learning and auditory training in cochlear implant recipients. Trends Amplif. 2007, 11, 193-205. [CrossRef]

6. Ingvalson, E.M.; Lee, B.; Fiebig, P.; Wong, P.C.M. The effects of short-Term computerized speech-In-Noise training on postlingually deafened adult cochlear implant recipients. J. Speech Lang. Hear. Res. 2013, 56, 81-88. [CrossRef]

7. Glennon, E.; Svirsky, M.A.; Froemke, R.C. Auditory cortical plasticity in cochlear implant users. Curr. Opin. Neurobiol. 2020, 60, 108-114. [CrossRef]

8. Chandramouli, S.H.; Kronenberger, W.G.; Pisoni, D.B. Verbal learning and memory in early-Implanted, prelingually deaf adolescent and adult cochlear implant users. J. Speech Lang. Hear. Res. 2019, 62, 1033-1050. [CrossRef]

9. Miller, S.; Zhang, Y.; Nelson, P. Neural correlates of phonetic learning in postlingually deafened cochlear implant listeners. Ear Hear. 2016, 37, 514-528. [CrossRef]

10. Chatterjee, M.; Zion, D.J.; Deroche, M.L.; Burianek, B.A.; Limb, C.J.; Goren, A.P.; Kulkarni, A.M.; Christensen, J.A. Voice emotion recognition by cochlear-implanted children and their normally-Hearing peers. Hear. Res. 2015, 322, 151-162. [CrossRef]

11. Jiam, N.T.; Caldwell, M.; Deroche, M.L.; Chatterjee, M.; Limb, C.J. Voice emotion perception and production in cochlear implant users. Hear. Res. 2017, 352, 30-39. [CrossRef] [PubMed]

12. Paquette, S.; Ahmed, G.D.; Goffi-Gomez, M.V.; Hoshino, A.C.H.; Peretz, I.; Lehmann, A. Musical and vocal emotion perception for cochlear implants users. Hear. Res. 2018, 370, 272-282. [CrossRef]

13. Chatterjee, M.; Peng, S.-C. Processing F0 with cochlear implants: Modulation frequency discrimination and speech intonation recognition. Hear. Res. 2008, 235, 143-156. [CrossRef]

14. Peng, S.-C.; Tomblin, J.B.; Turner, C.W. Production and perception of speech intonation in pediatric cochlear implant recipients and individuals with normal hearing. Ear Hear. 2008, 29, 336-351. [CrossRef] [PubMed]

15. Holt, C.M.; Demuth, K.; Yuen, I. The use of prosodic cues in sentence processing by prelingually deaf users of cochlear implants. Ear Hear. 2016, 37, e256-e262. [CrossRef]

16. Gu, X.; Liu, B.; Liu, Z.; Qi, B.; Wang, S.; Dong, R.; Chen, X.; Zhou, Q. A follow-Up study on music and lexical tone perception in adult Mandarin-Speaking cochlear implant users. Otol. Neurotol. 2017, 38, e421-e428. [CrossRef]

17. McDermott, H.J. Music perception with cochlear implants: A review. Trends Amplif. 2004, 8, 49-82. [CrossRef]

18. Tao, D.; Deng, R.; Jiang, Y.; Galvin III, J.J.; Fu, Q.-J.; Chen, B. Melodic pitch perception and lexical tone perception in Mandarin-Speaking cochlear implant users. Ear Hear. 2015, 36, 102-110. [CrossRef]

19. Holt, C.M.; Lee, K.Y.S.; Dowell, R.C.; Vogel, A.P. Perception of Cantonese lexical tones by pediatric cochlear implant users. J. Speech Lang. Hear. Res. 2018, 61, 174-185. [CrossRef]

20. Peng, S.-C.; Tomblin, J.B.; Cheung, H.; Lin, Y.-S.; Wang, L.-S. Perception and production of Mandarin tones in prelingually deaf children with cochlear implants. Ear Hear. 2004, 25, 251-264. [CrossRef] 
21. Peng, S.-C.; Lu, H.-P.; Lu, N.; Lin, Y.-S.; Deroche, M.L.D.; Chatterjee, M. Processing of acoustic cues in lexical-Tone identification by pediatric cochlear-implant recipients. J. Speech Lang. Hear. Res. 2017, 60, 1223-1235. [CrossRef] [PubMed]

22. Shpak, T.; Most, T.; Luntz, M. Fundamental frequency information for speech recognition via bimodal stimulation: Cochlear implant in one ear and hearing aid in the other. Ear Hear. 2014, 35, 97-109. [CrossRef]

23. Morera, C.; Manrique, M.; Ramos, A.; Garcia-Ibanez, L.; Cavalle, L.; Huarte, A.; Castillo, C.; Estrada, E. Advantages of binaural hearing provided through bimodal stimulation via a cochlear implant and a conventional hearing aid: A 6-Month comparative study. Acta Otolaryngol. 2005, 125, 596-606. [CrossRef] [PubMed]

24. Dorman, M.F.; Spahr, T.; Gifford, R.; Loiselle, L.; McKarns, S.; Holden, T.; Skinner, M.; Finley, C. An electric frequency-To-Place map for a cochlear implant patient with hearing in the nonimplanted ear. J. Assoc. Res. Otolaryngol. 2007, 8, 234-240. [CrossRef] [PubMed]

25. Mok, M.; Grayden, D.; Dowell, R.C.; Lawrence, D. Speech perception for adults who use hearing aids in conjunction with cochlear implants in opposite ears. J. Speech Lang. Hear. Res. 2006, 49, 338-351. [CrossRef]

26. Mok, M.; Galvin, K.L.; Dowell, R.C.; McKay, C.M. Speech perception benefit for children with a cochlear implant and a hearing aid in opposite ears and children with bilateral cochlear implants. Audiol. Neurotol. 2010, 15, 44-56. [CrossRef]

27. Cullington, H.E.; Zeng, F.-G. Comparison of bimodal and bilateral cochlear implant users on speech recognition with competing talker, music perception, affective prosody discrimination and talker identification. Ear Hear. 2011, 32, 16-30. [CrossRef]

28. Most, T.; Harel, T.; Shpak, T.; Luntz, M. Perception of suprasegmental speech features via bimodal stimulation: Cochlear implant on one ear and hearing aid on the other. J. Speech Lang. Hear. Res. 2011, 54, 668-678. [CrossRef]

29. Kong, Y.-Y.; Stickney, G.S.; Zeng, F.-G. Speech and melody recognition in binaurally combined acoustic and electric hearing. J. Acoust. Soc. Am. 2005, 117, 1351-1361. [CrossRef]

30. Zhang, T.; Spahr, A.J.; Dorman, M.F.; Saoji, A. The relationship between auditory function of non-implanted ears and bimodal benefit. Ear Hear. 2013, 34, 133-141. [CrossRef]

31. Chang, Y.; Chang, R.Y.; Lin, C.-Y.; Luo, X. Mandarin tone and vowel recognition in cochlear implant users: Effects of talker variability and bimodal hearing. Ear Hear. 2016, 37, 271-281. [CrossRef] [PubMed]

32. Li, Y.; Zhang, G.; Galvin III, J.J.; Fu, Q.-J. Mandarin speech perception in combined electric and acoustic stimulation. PLoS ONE 2014, 9, e112471. [CrossRef] [PubMed]

33. Luo, X.; Chang, Y.; Lin, C.-Y.; Chang, R.Y. Contribution of bimodal hearing to lexical tone normalization in Mandarin-Speaking cochlear implant users. Hear. Res. 2014, 312, 1-8. [CrossRef] [PubMed]

34. Mok, M.; Holt, C.M.; Lee, K.; Dowell, R.C.; Vogel, A.P. Cantonese tone perception for children who use a hearing aid and a cochlear implant in opposite ears. Ear Hear. 2017, 38, e359-e368. [CrossRef] [PubMed]

35. Yang, H.-I.; Zeng, F.-G. Bimodal benefits in Mandarin-Speaking cochlear implant users with contralateral residual acoustic hearing. Int. J. Audiol. 2017, 56, S17-S22. [CrossRef]

36. Yuen, K.C.P.; Cao, K.; Wei, C.; Luan, L.; Li, H.; Zhang, Z. Lexical tone and word recognition in noise of Mandarin-Speaking children who use cochlear implants and hearing aids in opposite ears. Cochlear Implant. Int. 2009, 10, 120-129. [CrossRef]

37. Chen, Y.; Wong, L.L.N.; Chen, F.; Xi, X. Tone and sentence perception in young Mandarin-speaking children with cochlear implants. Int. J. Pediatr. Otorhinolaryngol. 2014, 78, 1923-1930. [CrossRef]

38. Zhou, N.; Huang, J.; Chen, X.; Xu, L. Relationship between tone perception and production in prelingually-Deafened children with cochlear implants. Otol. Neurotol. 2013, 34, 499-509. [CrossRef]

39. Hong, T.; Wang, J.; Zhang, L.; Zhang, Y.; Shu, H.; Li, P. Age-Sensitive associations of segmental and suprasegmental perception with sentence-Level language skills in Mandarin-speaking children with cochlear implants. Res. Dev. Disabil. 2019, 93, 103453. [CrossRef]

40. Kral, A. Auditory critical periods: A review from system's perspective. Neuroscience 2013, 247, 117-133. [CrossRef]

41. Irvine, D.R.F. Plasticity in the auditory system. Hear. Res. 2018, 362, 61-73. [CrossRef] [PubMed]

42. Crew, J.D.; Galvin III, J.J.; Landsberger, D.M.; Fu, Q.-J. Contributions of electric and acoustic hearing to bimodal speech and music perception. PLOS ONE 2015, 10, e0120279. [CrossRef] [PubMed]

43. Hiskey, M.S. Hiskey-Nebraska Test of Learning Aptitude; Union College Press: Cambridge, UK, 1966. 
44. Boersma, P.; Weenink, D. Praat: Doing Phonetics by Computer (Computer Program, Version 6.0.33). Available online: http://www.fon.hum.uva.nl/praat/ (accessed on 26 September 2017).

45. Zhu, S.; Wong, L.L.N.; Chen, F. Development and validation of a new Mandarin tone identification test. Int. J. Pediatr. Otorhinolaryngol. 2014, 78, 2174-2182. [CrossRef] [PubMed]

46. Gifford, R.H.; Dorman, M.F. Bimodal hearing or bilateral cochlear implants? Ask the patient. Ear Hear. 2019, 40, 501-516. [CrossRef]

47. Studebaker, G.A. A "rationalized" arcsine transform. J. Speech Lang. Hear. Res. 1985, 28, 455-462. [CrossRef]

48. Zhang, L.; Wang, J.; Hong, T.; Li, Y.; Zhang, Y.; Shu, H. Mandarin-Speaking, kindergarten-Aged children with cochlear implants benefit from natural F0 patterns in the use of semantic context during speech recognition. J. Speech Lang. Hear. Res. 2018, 61, 2146-2152. [CrossRef]

49. Bates, D.; Mächler, M.; Bolker, B.M.; Walker, S.C. Fitting linear mixed-Effects models using lme4. J. Stat. Softw. 2015, 67, 1-48. [CrossRef]

50. Kuznetsova, A.; Brockhoff, P.B.; Christensen, R.H.B. lmerTest package: Tests in linear mixed effects models. J. Stat. Softw. 2017, 82, 1-26. [CrossRef]

51. Lenth, R.V. Least-Squares means: The R package lsmeans. J. Stat. Softw. 2016, 69, 1-33. [CrossRef]

52. Devocht, E.M.J.; Janssen, A.M.L.; Chalupper, J.; Stokroos, R.J.; George, E.L.J. The benefits of bimodal aiding on extended dimensions of speech perception: Intelligibility, listening effort, and sound quality. Trends Hear. 2017, 21. [CrossRef]

53. Gifford, R.H.; Stecker, G.C. Binaural cue sensitivity in cochlear implant recipients with acoustic hearing preservation. Hear. Res. 2020, 390, 107929. [CrossRef] [PubMed]

54. Hoppe, U.; Hocke, T.; Digeser, F. Bimodal benefit for cochlear implant listeners with different grades of hearing loss in the opposite ear. Acta Otolaryngol. 2018, 138, 713-721. [CrossRef] [PubMed]

55. Kessler, D.M.; Ananthakrishnan, S.; Smith, S.B.; D'Onofrio, K.; Gifford, R.H. Frequency following response and speech recognition benefit for combining a cochlear implant and contralateral hearing aid. Trends Hear. 2020, 24, 2331216520902001. [CrossRef]

56. Oh, S.H.; Donaldson, G.S.; Kong, Y.-Y. Top-Down processes in simulated electric-acoustic hearing: The effect of linguistic context on bimodal benefit for temporally interrupted speech. Ear Hear. 2016, 37, 582. [CrossRef]

57. Perreau, A.E.; Ou, H.; Tyler, R.; Dunn, C. Self-Reported spatial hearing abilities across different cochlear implant profiles. Am. J. Audiol. 2014, 23, 374-384. [CrossRef]

58. Shen, X.S.; Lin, M. A perceptual study of Mandarin tones 2 and 3. Lang. Speech 1991, 34, 145-156. [CrossRef]

59. Zhang, Y.; Wang, Y. Neural plasticity in speech acquisition and learning. Biling. Lang. Cogn. 2007, 10, 147-160. [CrossRef]

60. Chang, J.E.; Bai, J.Y.; Zeng, F.-G. Unintelligible low-Frequency sound enhances simulated cochlear-Implant speech recognition in noise. IEEE Trans. Biomed. Eng. 2006, 53, 2598-2601. [CrossRef]

61. Brown, C.A.; Bacon, S.P. Fundamental frequency and speech intelligibility in background noise. Hear. Res. 2010, 266, 52-59. [CrossRef]

62. Zhang, T.; Dorman, M.F.; Spahr, A.J. Information from the voice fundamental frequency (F0) region accounts for the majority of the benefit when acoustic stimulation is added to electric stimulation. Ear Hear. 2010, 31, 63-69. [CrossRef]

63. Li, N.; Loizou, P.C. A glimpsing account for the benefit of simulated combined acoustic and electric hearing. J. Acoust. Soc. Am. 2008, 123, 2287-2294. [CrossRef] [PubMed]

64. Kong, Y.-Y.; Carlyon, R.P. Improved speech recognition in noise in simulated binaurally combined acoustic and electric stimulation. J. Acoust. Soc. Am. 2007, 121, 3717-3727. [CrossRef] [PubMed]

65. Cooke, M. A glimpsing model of speech perception in noise. J. Acoust. Soc. Am. 2006, 119, 1562-1573. [CrossRef] [PubMed]

66. Easwar, V.; Yamazaki, H.; Deighton, M.; Papsin, B.; Gordon, K. Cortical representation of interaural time difference is impaired by deafness in development: Evidence from children with early long-term access to sound through bilateral cochlear implants provided simultaneously. J. Neurosci. 2017, 37, 2349-2361. [CrossRef]

67. Polonenko, M.J.; Papsin, B.C.; Gordon, K.A. Delayed access to bilateral input alters cortical organization in children with asymmetric hearing. NeuroImage Clin. 2018, 17, 415-425. [CrossRef]

68. Polonenko, M.J.; Papsin, B.C.; Gordon, K.A. Cortical plasticity with bimodal hearing in children with asymmetric hearing loss. Hear. Res. 2019, 372, 88-98. [CrossRef] 
69. Koerner, T.; Zhang, Y. Application of linear mixed-effects models in human neuroscience research: A comparison with Pearson correlation in two auditory electrophysiology studies. Brain Sci. 2017, 7, 26. [CrossRef]

70. Brungart, D.S. Informational and energetic masking effects in the perception of two simultaneous talkers. J. Acoust. Soc. Am. 2001, 109, 1101-1109. [CrossRef]

71. Neuman, A.C.; Waltzman, S.B.; Shapiro, W.H.; Neukam, J.D.; Zeman, A.M.; Svirsky, M.A. Self-Reported usage, functional benefit, and audiologic characteristics of cochlear implant patients who use a contralateral hearing aid. Trends Hear. 2017, 21, 1-14. [CrossRef]

72. Miller, S.E.; Zhang, Y.; Nelson, P.B. Efficacy of multiple-Talker phonetic identification training in postlingually deafened cochlear implant listeners. J. Speech Lang. Hear. Res. 2016, 59, 90-98. [CrossRef]

C 2020 by the authors. Licensee MDPI, Basel, Switzerland. This article is an open access article distributed under the terms and conditions of the Creative Commons Attribution (CC BY) license (http://creativecommons.org/licenses/by/4.0/). 